\title{
A relaxed hybrid shrinking iteration approach to solving generalized mixed equilibrium problems for totally quasi- $\phi$-asymptotically nonexpansive mappings
}

Wei-Qi Deng*

"Correspondence:

dwq1273@126.com

College of Statistics and

Mathematics, Yunnan University of

Finance and Economics, Kunming,

Yunnan 650221, P.R. China

\begin{abstract}
An optimal existing method for the approximation of common fixed points of countable families of nonlinear operators is introduced, by which a relaxed hybrid shrinking iterative algorithm is developed for the class of totally

quasi- $\phi$-asymptotically nonexpansive mappings, and a strong convergence theorem for solving generalized mixed equilibrium problems is established in the framework of Banach spaces. Since there is no need to impose the uniformity assumption on the involved countable family of mappings and no need to compute a complex series at each step in the iteration process, the result is more widely applicable than those of other authors with related interests.
\end{abstract}

MSC: 47J06; 47J25

Keywords: totally quasi- $\phi$-asymptotically nonexpansive mappings; generalized mixed equilibrium problems; generalized projections

\section{Introduction}

Throughout this paper we assume that $E$ is a real Banach space with its dual $E^{*}, C$ is a nonempty closed convex subset of $E$ and $J: E \rightarrow 2^{E^{*}}$ is the normalized duality mapping defined by

$$
J x=\left\{f \in E^{*}:\langle x, f\rangle=\|x\|^{2}=\|f\|^{2}\right\}, \quad \forall x \in E .
$$

In the sequel, we use $F(T)$ to denote the set of fixed points of a mapping $T$.

Definition 1.1 [1] (1) A mapping $T: C \rightarrow C$ is said to be totally quasi- $\phi$-asymptotically nonexpansive, if $F(T) \neq \emptyset$ and there exist nonnegative real sequences $\left\{v_{n}\right\},\left\{\mu_{n}\right\}$ with $v_{n}, \mu_{n} \rightarrow 0($ as $n \rightarrow \infty)$ and a strictly increasing continuous function $\zeta: \mathbb{R}^{+} \cup\{0\} \rightarrow$ $\mathbb{R}^{+} \cup\{0\}$ with $\zeta(0)=0$ such that

$$
\phi\left(p, T^{n} x\right) \leq \phi(p, x)+v_{n} \zeta(\phi(p, x))+\mu_{n}, \quad \forall n \geq 1, x \in C, p \in F(T),
$$

where $\phi: E \times E \rightarrow \mathbb{R}^{+} \cup\{0\}$ denotes the Lyapunov functional defined by

$$
\phi(x, y)=\|x\|^{2}-2\langle x, J y\rangle+\|y\|^{2}, \quad \forall x, y \in E .
$$

@2014 Deng; licensee Springer. This is an Open Access article distributed under the terms of the Creative Commons Attribution License (http://creativecommons.org/licenses/by/2.0), which permits unrestricted use, distribution, and reproduction in any medium, provided the original work is properly cited. 
It is obvious from the definition of $\phi$ that

$$
(\|x\|-\|y\|)^{2} \leq \phi(x, y) \leq(\|x\|+\|y\|)^{2}, \quad \forall x, y \in E
$$

and

$$
\phi\left(x, J^{-1}(\lambda J y+(1-\lambda) J z)\right) \leq \lambda \phi(x, y)+(1-\lambda) \phi(x, z), \quad \forall x, y \in E, \lambda \in[0,1] .
$$

(2) A countable family of mappings $\left\{T_{i}\right\}_{i=1}^{\infty}: C \rightarrow C$ said to be uniformly quasi- $\phi$ asymptotically nonexpansive, if $F:=\bigcap_{i=1}^{\infty} F\left(T_{i}\right) \neq \emptyset$ and there exists a nonnegative real sequence $\left\{k_{n}\right\} \subset[1, \infty)$ with $k_{n} \rightarrow 1($ as $n \rightarrow \infty)$ such that

$$
\phi\left(p, T_{i}^{n} x\right) \leq k_{n} \phi(p, x), \quad \forall n \geq 1, i \geq 1, x \in C, p \in F(T) .
$$

(3) A countable family of mappings $\left\{T_{i}\right\}_{i=1}^{\infty}: C \rightarrow C$ said to be uniformly totally quasi$\phi$-asymptotically nonexpansive, if $F:=\bigcap_{i=1}^{\infty} F\left(T_{i}\right) \neq \emptyset$ and there exist nonnegative real sequences $\left\{v_{n}\right\},\left\{\mu_{n}\right\}$ with $v_{n}, \mu_{n} \rightarrow 0$ (as $\left.n \rightarrow \infty\right)$ and a strictly increasing continuous function $\zeta: \mathbb{R}^{+} \cup\{0\} \rightarrow \mathbb{R}^{+} \cup\{0\}$ with $\zeta(0)=0$ such that

$$
\phi\left(p, T_{i}^{n} x\right) \leq \phi(p, x)+v_{n} \zeta(\phi(p, x))+\mu_{n}, \quad \forall n \geq 1, i \geq 1, x \in C, p \in F(T) .
$$

(4) A mapping $T: C \rightarrow C$ is said to be uniformly L-Lipschitz continuous, if there exists a constant $L>0$ such that

$$
\left\|T^{n} x-T^{n} y\right\| \leq L\|x-y\|, \quad \forall n \geq 1, x, y \in C .
$$

Let $\theta: C \times C \rightarrow \mathbb{R}$ be a bifunction, $\psi: C \rightarrow \mathbb{R}$ a real valued function and $A: C \rightarrow E^{*}$ a nonlinear mapping. The so-called generalized mixed equilibrium problem $(G M E P)$ is to find an $u \in C$ such that

$$
\theta(u, y)+\langle A u, y-u\rangle+\psi(y)-\psi(u) \geq 0, \quad \forall y \in C,
$$

whose set of solutions is denoted by $\Omega$. The equilibrium problem is an unifying model for several problems arising in physics, engineering, science optimization, economics, transportation, network and structural analysis, Nash equilibrium problems in noncooperative games, and others. It has been shown that variational inequalities and mathematical programming problems can be viewed as a special realization of the abstract equilibrium problems. Many authors have proposed some useful methods to solve the EP (equilibrium problem), GEP (generalized equilibrium problem), MEP (mixed equilibrium problem), and GMEP. Concerning the weak and strong convergence of iterative sequences to a common element of the set of solutions for the GMEP, the set of solutions for variational inequality problems, and the set of common fixed points for relatively nonexpansive mappings, quasi- $\phi$-nonexpansive mappings, quasi- $\phi$-asymptotically nonexpansive mappings and total quasi- $\phi$-asymptotically nonexpansive mappings have been studied by many authors in the setting of Hilbert or Banach spaces (see, for example, [2-17] and the references therein). 
In 2010, Qin et al. [18] proposed the following shrinking projection method to find a common element of the set of solutions of an equilibrium problem and the set of common fixed points of a finite family of quasi- $\phi$-nonexpansive mappings in the framework of Banach spaces:

$$
\left\{\begin{array}{l}
x_{0} \in C ; \quad C_{0}=C, \\
y_{n}=J^{-1}\left[\alpha_{n, 0} J x_{n}+\sum_{i=1}^{N} \alpha_{n, i} J T_{i} x_{n}\right], \\
u_{n} \in C \quad \text { such that } \forall y \in C, \\
\theta\left(u_{n}, y\right)+\frac{1}{r_{n}}\left\langle y-u_{n}, J u_{n}-J y_{n}\right\rangle \geq 0, \\
C_{n+1}=\left\{v \in C_{n}: \phi\left(v, u_{n}\right) \leq \phi\left(v, x_{n}\right)\right\}, \\
x_{n+1}=\prod_{C_{n+1}} x_{0}, \quad \forall n \geq 0,
\end{array}\right.
$$

where $\Pi_{C_{n+1}}$ is the generalized projection (see (2.1)) of $E$ onto $C_{n+1}$.

In 2011, Saewan and Kumam [19] introduced a modified new hybrid projection method to find a common element of the set of solutions of the generalized mixed equilibrium problems and the set of common fixed points of an infinite family of closed and uniformly quasi- $\phi$-asymptotically nonexpansive mappings in an uniformly smooth and strictly convex Banach spaces $E$ with the Kadec-Klee property:

$$
\left\{\begin{array}{l}
x_{0} \in C ; \quad C_{0}=C, \\
y_{n}=J^{-1}\left[\alpha_{n} J x_{n}+\left(1-\alpha_{n}\right) J z_{n}\right], \\
z_{n}=J^{-1}\left[\beta_{n, 0} J x_{n}+\sum_{i=1}^{\infty} \beta_{n, i} J T_{i}^{n} x_{n}\right], \\
u_{n} \in C \quad \text { such that } u_{n}=K_{r_{n}} y_{n}, \\
C_{n+1}=\left\{z \in C_{n}: \phi\left(z, u_{n}\right) \leq \phi\left(z, x_{n}\right)+\xi_{n}\right\}, \\
x_{n+1}=\prod_{C_{n+1}} x_{0}, \quad \forall n \geq 0,
\end{array}\right.
$$

where $\xi_{n}:=\left(k_{n}-1\right) \sup _{p \in F} \zeta\left(\phi\left(p, x_{n}\right)\right)$.

However, it is obviously a quite strong condition that the involved mappings are assumed to be a countable family of uniformly $\left(\left\{v_{n}\right\},\left\{\mu_{n}\right\}, \zeta\right)$-quasi- $\phi$-asymptotically nonexpansive ones, which is a special case of totally quasi- $\phi$-asymptotically nonexpansive mappings (see [10]). In addition, the accurate computation of the series $\sum_{i=1}^{\infty} \beta_{n, i} J T_{i}^{n} y_{n}$ at each step of the iteration process is not easily attainable, which will lead to gradually increasing errors.

Inspired and motivated by the studies mentioned above, by using a special way of choosing the indices, we propose a relaxed hybrid shrinking iteration scheme for approximating common fixed points of a countable family of totally quasi- $\phi$-asymptotically nonexpansive mappings and obtain a strong convergence theorem for solving the generalized mixed equilibrium problems under suitable conditions, namely, there is no need to assume uniformity for the totally quasi- $\phi$-asymptotic property of the involved mappings, and no need to compute complex series in the iteration process. The results extend and improve those of other authors with related interests.

\section{Preliminaries}

We say that a Banach space $E$ is strictly convex if the following implication holds for $x, y \in E$ :

$$
\|x\|=\|y\|=1, \quad x \neq y \quad \Rightarrow \quad\left\|\frac{x+y}{2}\right\|<1 .
$$


It is also said to be uniformly convex if for any $\epsilon>0$, there exists a $\delta>0$ such that

$$
\|x\|=\|y\|=1, \quad\|x-y\| \geq \epsilon \quad \Rightarrow \quad\left\|\frac{x+y}{2}\right\| \leq 1-\delta .
$$

It is well known that if $E$ is a uniformly convex Banach space, then $E$ is reflexive and strictly convex. A Banach space $E$ is said to be smooth if

$$
\lim _{t \rightarrow 0} \frac{\|x+t y\|-\|x\|}{t}
$$

exists for each $x, y \in S(E):=\{x \in E:\|x\|=1\}$. E is said to be uniformly smooth if the limit (2.3) is attained uniformly for $x, y \in S(E)$.

Following Alber [20], the generalized projection $\Pi_{C}: E \rightarrow C$ is defined by

$$
\Pi_{C}=\arg \inf _{y \in C} \phi(y, x), \quad \forall x \in E .
$$

Lemma 2.1 [20] Let E be a smooth, strictly convex and reflexive Banach space and C be a nonempty closed convex subset of $E$. Then the following conclusions hold:

(1) $\phi\left(x, \Pi_{C} y\right)+\phi\left(\Pi_{C} y, y\right) \leq \phi(x, y)$ for all $x \in C$ and $y \in E$;

(2) If $x \in E$ and $z \in C$, then $z=\Pi_{C} x \Leftrightarrow\langle z-y, J x-J z\rangle \geq 0, \forall y \in C$;

(3) For $x, y \in E, \phi(x, y)=0$ if and only if $x=y$.

Remark 2.2 The following basic properties for a Banach space $E$ can be found in Cioranescu [21].

(i) If $E$ is uniformly smooth, then $J$ is uniformly continuous on each bounded subset of $E$;

(ii) If $E$ is reflexive and strictly convex, then $J^{-1}$ is norm-weak-continuous;

(iii) If $E$ is reflexive smooth and strictly convex, then the normalized duality mapping $J$ is single-valued, one-to-one and onto;

(iv) A Banach space $E$ is uniformly smooth if and only if $E^{*}$ is uniformly convex;

(v) Each uniformly convex Banach space $E$ has the Kadec-Klee property, i.e., for any sequence $\left\{x_{n}\right\} \subset E$, if $x_{n} \rightarrow x \in E$ and $\left\|x_{n}\right\| \rightarrow\|x\|$, then $x_{n} \rightarrow x$ as $n \rightarrow \infty$.

Lemma 2.3 [22] Let E be a real uniformly smooth and strictly convex Banach space with Kadec-Klee property, and C be a nonempty closed convex subset of E. Let $\left\{x_{n}\right\}$ and $\left\{y_{n}\right\}$ be two sequences in $C$ such that $x_{n} \rightarrow p$ and $\phi\left(x_{n}, y_{n}\right) \rightarrow 0$, where $\phi$ is the function defined by (1.2), then $y_{n} \rightarrow p$.

Lemma 2.4 [22] Let $E$ and $C$ be the same as in Lemma 2.3. Let $T: C \rightarrow C$ be a closed and totally quasi- $\phi$-asymptotically nonexpansive mappings with nonnegative real sequences $\left\{v_{n}\right\},\left\{\mu_{n}\right\}$ and a strictly increasing continuous function $\zeta: \mathbb{R}^{+} \cup\{0\} \rightarrow \mathbb{R}^{+} \cup\{0\}$ such that $v_{n}, \mu_{n} \rightarrow 0$ and $\zeta(0)=0$. If $\mu_{1}=0$, then the fixed point set $F(T)$ of $T$ is a closed and convex subset of $C$.

Lemma 2.5 [1] Let $E$ be a real uniformly convex Banach space and let $B_{r}(0)$ be the closed ball of $E$ with center at the origin and radius $r>0$. Then for any for any sequence $\left\{x_{i}\right\} \subset B_{r}(0)$ and for any sequence $\left\{\lambda_{i}\right\}$ of positive numbers with $\sum_{i=1}^{\infty} \lambda_{i}=1$, there exists a 
continuous strictly increasing convex function $g:[0, \infty) \rightarrow[0, \infty)$ with $g(0)=0$ such that such that for any positive integer $i \neq 1$, the following hold:

$$
\left\|\sum_{i=1}^{\infty} \lambda_{i} x_{i}\right\|^{2} \leq \sum_{i=1}^{\infty} \lambda_{i}\left\|x_{i}\right\|^{2}-\lambda_{1} \lambda_{i} g\left(\left\|x_{1}-x_{i}\right\|\right),
$$

and for all $x \in E$,

$$
\phi\left(x, J^{-1}\left(\sum_{i=1}^{\infty} \lambda_{j} J x_{i}\right)\right) \leq \sum_{i=1}^{\infty} \lambda_{i} \phi\left(x, x_{i}\right)-\lambda_{1} \lambda_{i} g\left(\left\|J x_{1}-J x_{i}\right\|\right) .
$$

Assume that, to obtain the solution of GMEP, the function $\psi: C \rightarrow \mathbb{R}$ is convex and lower semicontinuous, the nonlinear mapping $A: C \rightarrow E^{*}$ is continuous and monotone, and the bifunction $\theta: C \times C \rightarrow \mathbb{R}$ satisfies the following conditions:

$\left(\mathrm{A}_{1}\right) \theta(x, x)=0$

$\left(\mathrm{A}_{2}\right) \theta$ is monotone, i.e., $\theta(x, y)+\theta(y, x) \leq 0$;

$\left(\mathrm{A}_{3}\right) \lim \sup _{t \downarrow 0} \theta(x+t(z-x), y) \leq \theta(x, y)$;

$\left(\mathrm{A}_{4}\right)$ the mapping $y \mapsto \theta(x, y)$ is convex and lower semicontinuous.

Lemma 2.6 [15] Let $E$ be a smooth, strictly convex, and reflexive Banach space, and $C$ be a nonempty closed convex subset of $E$. Let $A: C \rightarrow E^{*}$ be a continuous and monotone mapping, $\psi: C \rightarrow \mathbb{R}$ a lower semicontinuous and convex function, and $\theta: C \times C \rightarrow \mathbb{R}$ a bifunction satisfying the conditions $\left(\mathrm{A}_{1}\right)-\left(\mathrm{A}_{4}\right)$. Let $r>0$ and $x \in E$. Then, the following hold:

(1) There exists an $u \in C$ such that

$$
\theta(u, y)+\langle A u, y-u\rangle+\psi(y)-\psi(u)+\frac{1}{r}\langle y-u, J u-J x\rangle \geq 0, \quad \forall y \in C .
$$

(2) A mapping $\kappa_{r}: C \rightarrow C$ is defined by

$$
\kappa_{r}(x)=\left\{u \in C: \theta(u, y)+\langle A u, y-u\rangle+\psi(y)-\psi(u)+\frac{1}{r}\langle y-u, J u-J x\rangle \geq 0, \forall y \in C\right\} .
$$

Then, the mapping $\kappa_{r}$ has the following properties:

(i) $\kappa_{r}$ is single-valued;

(ii) $\kappa_{r}$ a firmly nonexpansive-type mapping, i.e.,

$$
\left\langle\kappa_{r} z-\kappa_{r} y, J \kappa_{r} z-J \kappa_{r} y\right\rangle \leq\left\langle\kappa_{r} z-\kappa_{r} y, J z-J y\right\rangle
$$

(iii) $F\left(\kappa_{r}\right)=\Omega=\tilde{F}\left(\kappa_{r}\right)$;

(iv) $\Omega$ is a closed convex set of $C$;

(v) $\phi\left(p, \kappa_{r} z\right)+\phi\left(\kappa_{r} z, z\right) \leq \phi(p, z), \forall p \in F\left(\kappa_{r}\right), z \in E$,

where $\tilde{F}\left(\kappa_{r}\right)$ denotes the set of asymptotic fixed points of $\kappa_{r}$, i.e.,

$$
\tilde{F}\left(\kappa_{r}\right):=\left\{x \in C: \exists\left\{x_{n}\right\} \subset C \text {, s.t. }, x_{n} \rightarrow x,\left\|x_{n}-\kappa_{r} x_{n}\right\| \rightarrow 0(n \rightarrow \infty)\right\} .
$$


Lemma 2.7 [23] The unique solutions to the positive integer equation

$$
n=i+\frac{(m-1) m}{2}, \quad m \geq i, n=1,2, \ldots
$$

are

$$
i=n-\frac{(m-1) m}{2}, \quad m=-\left[\frac{1}{2}-\sqrt{2 n+\frac{1}{4}}\right], \quad n=1,2, \ldots
$$

where $[x]$ denotes the maximal integer that is not larger than $x$.

\section{Main results}

Recall that a mapping $T$ on a Banach space is closed if $x_{n} \rightarrow x$ and $T x_{n} \rightarrow y$ as $n \rightarrow \infty$, then $T x=y$.

Theorem 3.1 Let $E$ be a real uniformly smooth and strictly convex Banach space with Kadec-Klee property and $C$ a nonempty closed convex subset of $E$. Let $\theta: C \times C \rightarrow \mathbb{R}$ be a bifunction satisfying the conditions $\left(\mathrm{A}_{1}\right)-\left(\mathrm{A}_{4}\right), A: C \rightarrow E^{*}$ a continuous and monotone mapping, and $\psi: C \rightarrow \mathbb{R}$ a lower semicontinuous and convex function. Let $\left\{T_{i}\right\}_{i=1}^{\infty}: C \rightarrow C$ be a countable family of closed and totally quasi- $\phi$-asymptotically nonexpansive mappings with nonnegative real sequences $\left\{v_{n}^{(i)}\right\},\left\{\mu_{n}^{(i)}\right\}$ satisfying $v_{n}^{(i)} \rightarrow 0$ and $\mu_{n}^{(i)} \rightarrow 0($ as $n \rightarrow \infty$ and for each $i \geq 1)$ and a sequence of strictly increasing and continuous functions $\left\{\zeta_{i}\right\}$ : $\mathbb{R}^{+} \cup\{0\} \rightarrow \mathbb{R}^{+} \cup\{0\}$ satisfying condition (1.1). Assume that each $T_{i}$ is uniformly $L_{i}$-Lipschitz continuous with $\mu_{1}^{(i)}=0$ for each $i \geq 1$. Let $\left\{\alpha_{n}\right\}$ be a sequence in $[0, \epsilon]$ for some $\epsilon \in(0,1)$ and $\left\{\beta_{i}\right\}$ be a sequence in $(0,1)$. Let $\left\{x_{n}\right\}$ be the sequence generated by

$$
\left\{\begin{array}{l}
x_{1} \in C ; \quad C_{1}=C, \\
y_{n}=J^{-1}\left[\alpha_{n} J x_{n}+\left(1-\alpha_{n}\right) J z_{n}\right], \\
z_{n}=J^{-1}\left[\beta_{i_{n}} J x_{n}+\left(1-\beta_{i_{n}}\right) J T_{i_{n}}^{m_{n}} x_{n}\right], \\
u_{n} \in C \quad \text { such that } \forall y \in C, \\
\theta\left(u_{n}, y\right)+\left\langle A u_{n}, y-u_{n}\right\rangle+\psi(y)-\psi\left(u_{n}\right)+\frac{1}{r_{n}}\left\langle y-u_{n}, J u_{n}-J y_{n}\right\rangle \geq 0, \\
C_{n+1}=\left\{v \in C_{n}: \phi\left(v, u_{n}\right) \leq \phi\left(v, x_{n}\right)+\xi_{n}\right\}, \\
x_{n+1}=\prod_{C_{n+1}} x_{1}, \quad n \in \mathbb{N},
\end{array}\right.
$$

where $\xi_{n}:=v_{m_{n}}^{\left(i_{n}\right)} \sup _{p \in F} \zeta_{i_{n}}\left(\phi\left(p, x_{n}\right)\right)+\mu_{m_{n}}^{\left(i_{n}\right)}$ and $\Pi_{C_{n+1}}$ is the generalized projection of $E$ onto $C_{n+1} ; i_{n}$ and $m_{n}$ are the solutions to the positive integer equation: $n=i+\frac{(m-1) m}{2}(m \geq i$, $n=1,2, \ldots)$, that is, for each $n \geq 1$, there exist unique $i_{n}$ and $m_{n}$ such that

$$
\begin{array}{ll}
i_{1}=1, & i_{2}=1, \quad i_{3}=2, \quad i_{4}=1, \\
i_{5}=2, & i_{6}=3, \quad i_{7}=1, \quad i_{8}=2, \ldots ; \\
m_{1}=1, & m_{2}=2, \quad m_{3}=2, \quad m_{4}=3, \\
m_{5}=3, & m_{6}=3, \quad m_{7}=4, \quad m_{8}=4, \ldots
\end{array}
$$

If $G:=F \cap \Omega \neq \emptyset$ and $F:=\bigcap_{i=1}^{\infty} F\left(T_{i}\right)$ is bounded, then $\left\{x_{n}\right\}$ converges strongly to $\Pi_{G} x_{1}$. 
Proof Two functions $\tau: C \times C \rightarrow \mathbb{R}$ and $\kappa_{r}: C \rightarrow C$ are defined by

$$
\begin{aligned}
& \tau(x, y)=\theta(x, y)+\langle A x, y-x\rangle+\psi(y)-\psi(x) \\
& \kappa_{r}(x)=\left\{u \in C: \tau(u, y)+\frac{1}{r}\langle y-u, J u-J x\rangle \geq 0, \forall y \in C\right\} .
\end{aligned}
$$

By Lemma 2.6, we know that the function $\tau$ satisfies the conditions $\left(\mathrm{A}_{1}\right)-\left(\mathrm{A}_{4}\right)$ and $\kappa_{r}$ has the property (i)-(v). Therefore, (3.1) can be rewritten as

$$
\left\{\begin{array}{l}
x_{1} \in C ; \quad C_{1}=C, \\
y_{n}=J^{-1}\left[\alpha_{n} J x_{n}+\left(1-\alpha_{n}\right) J z_{n}\right], \\
z_{n}=J^{-1}\left[\beta_{i_{n}} J x_{n}+\left(1-\beta_{i_{n}}\right) J T_{i_{n}}^{m_{n}} x_{n}\right], \\
u_{n} \in C \quad \text { such that } \tau\left(u_{n}, y\right)+\frac{1}{r_{n}}\left\langle y-u_{n}, J u_{n}-J y_{n}\right\rangle \geq 0, \forall y \in C, \\
C_{n+1}=\left\{v \in C_{n}: \phi\left(v, u_{n}\right) \leq \phi\left(v, x_{n}\right)+\xi_{n}\right\}, \\
x_{n+1}=\Pi_{C_{n+1}} x_{1}, \quad \forall n \geq 1 .
\end{array}\right.
$$

We divide the proof into several steps.

(I) $F$ and $C_{n}(\forall n \geq 1)$ both are closed and convex subsets in $C$.

In fact, it follows from Lemma 2.4 that each $F\left(T_{i}\right)$ is a closed and convex subset of $C$, so is $F$. In addition, with $C_{1}(=C)$ being closed and convex, we may assume that $C_{n}$ is closed and convex for some $n \geq 2$. In view of the definition of $\phi$ we have

$$
C_{n+1}=\{v \in C: \varphi(v) \leq a\} \cap C_{n}
$$

where $\varphi(v)=2\left\langle v, J x_{n}-J y_{n}\right\rangle$ and $a=\left\|x_{n}\right\|^{2}-\left\|y_{n}\right\|^{2}+\xi_{n}$. This shows that $C_{n+1}$ is closed and convex.

(II) $G$ is a subset of $\bigcap_{n=1}^{\infty} C_{n}$.

It is obvious that $G \subset C_{1}$. Suppose that $G \subset C_{n}$ for some $n \geq 2$. Since $u_{n}=\kappa_{r_{n}} y_{n}$, by Lemma 2.6, it is easily shown that $\kappa_{r_{n}}$ is quasi- $\phi$-nonexpansive. Hence, for any $p \in G \subset C_{n}$, it follows from (1.4) that

$$
\begin{aligned}
\phi\left(p, u_{n}\right) & =\phi\left(p, \kappa_{r_{n}} y_{n}\right) \leq \phi\left(p, y_{n}\right) \\
& =\phi\left(p, J^{-1}\left[\alpha_{n} J x_{n}+\left(1-\alpha_{n}\right) J z_{n}\right]\right) \\
& \leq \alpha_{n} \phi\left(p, x_{n}\right)+\left(1-\alpha_{n}\right) \phi\left(p, z_{n}\right) .
\end{aligned}
$$

Furthermore, it follows from Lemma 2.5 that for any $p \in G \subset C_{n}$,

$$
\begin{aligned}
\phi\left(p, z_{n}\right)= & \phi\left(p, J^{-1}\left[\beta_{i_{n}} J x_{n}+\left(1-\beta_{i_{n}}\right) J T_{i_{n}}^{m_{n}} x_{n}\right]\right) \\
\leq & \beta_{i_{n}} \phi\left(p, x_{n}\right)+\left(1-\beta_{i_{n}}\right) \phi\left(p, T_{i_{n}}^{m_{n}} x_{n}\right)-\beta_{i_{n}}\left(1-\beta_{i_{n}}\right) g\left(\left\|J x_{n}-J T_{i_{n}}^{m_{n}} x_{n}\right\|\right) \\
\leq & \beta_{i_{n}} \phi\left(p, x_{n}\right)+\left(1-\beta_{i_{n}}\right)\left[\phi\left(p, x_{n}\right)+v_{m_{n}}^{\left(i_{n}\right)} \zeta_{i_{n}}\left(\phi\left(p, x_{n}\right)\right)+\mu_{m_{n}}^{\left(i_{n}\right)}\right] \\
& -\beta_{i_{n}}\left(1-\beta_{i_{n}}\right) g\left(\left\|J x_{n}-J T_{i_{n}}^{m_{n}} x_{n}\right\|\right) \\
\leq & \phi\left(p, x_{n}\right)+v_{m_{n}}^{\left(i_{n}\right)} \sup _{p \in F} \zeta_{i_{n}}\left(\phi\left(p, x_{n}\right)\right)+\mu_{m_{n}}^{\left(i_{n}\right)}-\beta_{i_{n}}\left(1-\beta_{i_{n}}\right) g\left(\left\|J x_{n}-J T_{i_{n}}^{m_{n}} x_{n}\right\|\right) \\
= & \phi\left(p, x_{n}\right)+\xi_{n}-\beta_{i_{n}}\left(1-\beta_{i_{n}}\right) g\left(\left\|J x_{n}-J T_{i_{n}}^{m_{n}} x_{n}\right\|\right) .
\end{aligned}
$$


Substituting (3.4) into (3.3) and simplifying it, we have

$$
\begin{aligned}
\phi\left(p, u_{n}\right) & \leq \phi\left(p, y_{n}\right) \leq \phi\left(p, x_{n}\right)+\left(1-\alpha_{n}\right) \xi_{n}-\left(1-\alpha_{n}\right) \beta_{i_{n}}\left(1-\beta_{i_{n}}\right) g\left(\left\|J x_{n}-J T_{i_{n}}^{m_{n}} x_{n}\right\|\right) \\
& \leq \phi\left(p, x_{n}\right)+\xi_{n}-\left(1-\alpha_{n}\right) \beta_{i_{n}}\left(1-\beta_{i_{n}}\right) g\left(\left\|J x_{n}-J T_{i_{n}}^{m_{n}} x_{n}\right\|\right) \\
& \leq \phi\left(p, x_{n}\right)+\xi_{n} .
\end{aligned}
$$

This implies that $p \in C_{n+1}$, and so $G \subset C_{n+1}$.

(III) $x_{n} \rightarrow x^{*} \in C$ as $n \rightarrow \infty$.

In fact, since $x_{n}=\Pi_{C_{n}} x_{1}$, from Lemma 2.1(2) we have $\left\langle x_{n}-y, J x_{1}-J x_{n}\right\rangle \geq 0, \forall y \in C_{n}$. Again since $F \subset \bigcap_{n=1}^{\infty} C_{n}$, we have $\left\langle x_{n}-p, J x_{1}-J x_{n}\right\rangle \geq 0, \forall p \in F$. It follows from Lemma 2.1(1) that for each $p \in F$ and for each $n \geq 1$,

$$
\phi\left(x_{n}, x_{1}\right)=\phi\left(\Pi_{C_{n}} x_{1}, x_{1}\right) \leq \phi\left(p, x_{1}\right)-\phi\left(p, x_{n}\right) \leq \phi\left(p, x_{1}\right)
$$

which implies that $\left\{\phi\left(x_{n}, x_{1}\right)\right\}$ is bounded, so is $\left\{x_{n}\right\}$. Since for all $n \geq 1, x_{n}=\Pi_{C_{n}} x_{1}$ and $x_{n+1}=\Pi_{C_{n+1}} x_{1} \in C_{n+1} \subset C_{n}$, we have $\phi\left(x_{n}, x_{1}\right) \leq \phi\left(x_{n+1}, x_{1}\right)$. This implies that $\left\{\phi\left(x_{n}, x_{1}\right)\right\}$ is nondecreasing, hence the limit

$$
\lim _{n \rightarrow \infty} \phi\left(x_{n}, x_{1}\right) \text { exists. }
$$

Since $E$ is reflexive, there exists a subsequence $\left\{x_{n_{i}}\right\}$ of $\left\{x_{n}\right\}$ such that $x_{n_{i}} \rightarrow x^{*} \in C$ as $i \rightarrow \infty$. Since $C_{n}$ is closed and convex and $C_{n+1} \subset C_{n}$, this implies that $C_{n}$ is weakly closed and $x^{*} \in C_{n}$ for each $n \geq 1$. In view of $x_{n_{i}}=\Pi_{C_{n_{i}}} x_{1}$, we have

$$
\phi\left(x_{n_{i}}, x_{1}\right) \leq \phi\left(x^{*}, x_{1}\right), \quad \forall i \geq 1
$$

Since the norm $\|\cdot\|$ is weakly lower semicontinuous, we have

$$
\begin{aligned}
\liminf _{i \rightarrow \infty} \phi\left(x_{n_{i}}, x_{1}\right) & =\liminf _{i \rightarrow \infty}\left(\left\|x_{n_{i}}\right\|^{2}-2\left\langle x_{n_{i}}, J x_{1}\right\rangle+\left\|x_{1}\right\|^{2}\right) \\
& \geq\left\|x^{*}\right\|^{2}-2\left\langle x^{*}, J x_{1}\right\rangle+\left\|x_{1}\right\|^{2} \\
& =\phi\left(x^{*}, x_{1}\right),
\end{aligned}
$$

and so

$$
\phi\left(x^{*}, x_{1}\right) \leq \liminf _{i \rightarrow \infty} \phi\left(x_{n_{i}}, x_{1}\right) \leq \limsup _{i \rightarrow \infty} \phi\left(x_{n_{i}}, x_{1}\right) \leq \phi\left(x^{*}, x_{1}\right)
$$

This implies that $\lim _{i \rightarrow \infty} \phi\left(x_{n_{i}}, x_{1}\right)=\phi\left(x^{*}, x_{1}\right)$, and so $\left\|x_{n_{i}}\right\| \rightarrow\left\|x^{*}\right\|$ as $i \rightarrow \infty$. Since $x_{n_{i}} \rightarrow$ $x^{*}$, by virtue of the Kadec-Klee property of $E$, we obtain

$$
\lim _{i \rightarrow \infty} x_{n_{i}}=x^{*}
$$

Since $\left\{\phi\left(x_{n}, x_{1}\right)\right\}$ is convergent, this, together with $\lim _{i \rightarrow \infty} \phi\left(x_{n_{i}}, x_{1}\right)=\phi\left(x^{*}, x_{1}\right)$, shows that $\lim _{n \rightarrow \infty} \phi\left(x_{n}, x_{1}\right)=\phi\left(x^{*}, x_{1}\right)$. If there exists some subsequence $\left\{x_{n_{j}}\right\}$ of $\left\{x_{n}\right\}$ such that $x_{n_{j}} \rightarrow$ 
$y$ as $j \rightarrow \infty$, then from Lemma 2.1(1) we have

$$
\begin{aligned}
\phi\left(x^{*}, y\right) & =\lim _{i, j \rightarrow \infty} \phi\left(x_{n_{i}}, x_{n_{j}}\right)=\lim _{i, j \rightarrow \infty} \phi\left(x_{n_{i}}, \Pi_{C_{n_{j}}} x_{1}\right) \\
& \leq \lim _{i, j \rightarrow \infty}\left(\phi\left(x_{n_{i}}, x_{1}\right)-\phi\left(\Pi_{C_{n_{j}}} x_{1}, x_{1}\right)\right) \\
& =\lim _{i, j \rightarrow \infty}\left(\phi\left(x_{n_{i}}, x_{1}\right)-\phi\left(x_{n_{j}}, x_{1}\right)\right) \\
& =\phi\left(x^{*}, x_{1}\right)-\phi\left(x^{*}, x_{1}\right)=0,
\end{aligned}
$$

that is, $x^{*}=y$ and so

$$
\lim _{n \rightarrow \infty} x_{n}=x^{*}
$$

(IV) $x^{*}$ is a member of $F$.

Set $\mathcal{K}_{i}=\left\{k \in \mathbb{N}: k=i+\frac{(m-1) m}{2}, m \geq i, m \in \mathbb{N}\right\}$ for each $i \in \mathbb{N}$. Note that $v_{m_{k}}^{\left(i_{k}\right)}=v_{m_{k}}^{(i)}$, $\mu_{m_{k}}^{\left(i_{k}\right)}=\mu_{m_{k}}^{(i)}$ and $\zeta_{i_{k}}=\zeta_{i}$ whenever $k \in \mathcal{K}_{i}$ for each $i \geq 1$. For example, by Lemma 2.7 and the definition of $\mathcal{K}_{1}$, we have $\mathcal{K}_{1}=\{1,2,4,7,11,16, \ldots\}$ and $i_{1}=i_{2}=i_{4}=i_{7}=i_{11}=i_{16}=\cdots=1$. Then we have

$$
\xi_{k}=v_{m_{k}}^{(i)} \sup _{p \in F} \zeta_{i}\left(\phi\left(p, x_{k}\right)\right)+\mu_{m_{k}}^{(i)}, \quad \forall k \in \mathcal{K}_{i}
$$

Note that $\left\{m_{k}\right\}_{k \in \mathcal{K}_{i}}=\{i, i+1, i+2, \ldots\}$, i.e., $m_{k} \uparrow \infty$ as $\mathcal{K}_{i} \ni k \rightarrow \infty$. It follows from (3.6) and (3.7) that

$$
\lim _{\mathcal{K}_{i \ni k \rightarrow \infty}} \xi_{k}=0, \quad \forall i \geq 1
$$

Since $x_{n+1} \in C_{n+1}$, it follows from (3.1), (3.6), and (3.8) that

$$
\phi\left(x_{k+1}, y_{k}\right) \leq \phi\left(x_{k+1}, x_{k}\right)+\xi_{k} \rightarrow 0 \quad\left(\mathcal{K}_{i} \ni k \rightarrow \infty\right)
$$

Since $x_{k} \rightarrow x^{*}$ as $\mathcal{K}_{i} \ni k \rightarrow \infty$, it follows from (3.9) and Lemma 2.3 that

$$
\lim _{\mathcal{K}_{i} \ni k \rightarrow \infty} y_{k}=x^{*}, \quad \forall i \geq 1
$$

Note that $T_{i_{k}}^{m_{k}}=T_{i}^{m_{k}}$ and $\beta_{i_{k}}=\beta_{i}$ whenever $k \in \mathcal{K}_{i}$ for each $i \geq 1$. From (3.5), for any $p \in F$, we have

$$
\phi\left(p, y_{k}\right) \leq \phi\left(p, x_{k}\right)+\xi_{k}-\left(1-\alpha_{k}\right) \beta_{i}\left(1-\beta_{i}\right) g\left(\left\|J x_{k}-J T_{i}^{m_{k}} x_{k}\right\|\right), \quad \forall k \in \mathcal{K}_{i},
$$

that is,

$$
\left(1-\alpha_{k}\right) \beta_{i}\left(1-\beta_{i}\right) g\left(\left\|J x_{k}-J T_{i}^{m_{k}} x_{k}\right\|\right) \leq \phi\left(p, x_{k}\right)+\xi_{k}-\phi\left(p, y_{k}\right) \rightarrow 0 \quad\left(\mathcal{K}_{i} \ni k \rightarrow \infty\right)
$$

This shows that $\lim _{\mathcal{K}_{i} \ni k \rightarrow \infty} g\left(\left\|J x_{k}-J T_{i}^{m_{k}} x_{k}\right\|\right)=0$. In view of the property of $g$, we have

$$
\lim _{\mathcal{K}_{i} \ni k \rightarrow \infty}\left\|J x_{k}-J T_{i}^{m_{k}} x_{k}\right\|=0, \quad \forall i \geq 1
$$


In addition, $J x_{k} \rightarrow J x^{*}\left(\mathcal{K}_{i} \ni k \rightarrow \infty\right)$ implies that $\lim _{\mathcal{K}_{i} \ni k \rightarrow \infty} J T_{i}^{m_{k}} x_{k}=J x^{*}$. From Remark 2.2(ii) it yields that, as $\mathcal{K}_{i} \ni k \rightarrow \infty$,

$$
T_{i}^{m_{k}} x_{k} \rightarrow x^{*}, \quad \forall i \geq 1
$$

Again since for each $i \geq 1$, as $\mathcal{K}_{i} \ni k \rightarrow \infty$,

$$
\left|\left\|T_{i}^{m_{k}} x_{k}\right\|-\left\|x^{*}\right\|\right|=\left|\left\|J T_{i}^{m_{k}} x_{k}\right\|-\left\|J x^{*}\right\|\right| \leq\left\|J T_{i}^{m_{k}} x_{k}-J x^{*}\right\| \rightarrow 0
$$

this, together with (3.11) and the Kadec-Klee property of $E$, shows that

$$
\lim _{\mathcal{K}_{i} \ni \rightarrow \infty} T_{i}^{m_{k}} x_{k}=x^{*}, \quad \forall i \geq 1
$$

We use the assumptions that for each $i \geq 1, T_{i}$ is uniformly $L_{i}$-Lipschitz continuous. Noting again that $\left\{m_{k}\right\}_{k \in \mathcal{K}_{i}}=\{i, i+1, i+2, \ldots\}$, i.e., $m_{k+1}-1=m_{k}$ for all $k \in \mathcal{K}_{i}$, we then have

$$
\begin{aligned}
\left\|T_{i}^{m_{k+1}} x_{k}-T_{i}^{m_{k}} x_{k}\right\| \leq & \left\|T_{i}^{m_{k+1}} x_{k}-T_{i}^{m_{k+1}} x_{k+1}\right\|+\left\|T_{i}^{m_{k+1}} x_{k+1}-x_{k+1}\right\| \\
& +\left\|x_{k+1}-x_{k}\right\|+\left\|x_{k}-T_{i}^{m_{k}} x_{k}\right\| \\
\leq & \left(L_{i}+1\right)\left\|x_{k+1}-x_{k}\right\|+\left\|T_{i}^{m_{k+1}} x_{k+1}-x_{k+1}\right\| \\
& +\left\|x_{k}-T_{i}^{m_{k}} x_{k}\right\| .
\end{aligned}
$$

From (3.12) and $x_{k} \rightarrow x^{*}$ as $\mathcal{K}_{i} \ni k \rightarrow \infty$ we have $\lim _{\mathcal{K}_{i} \ni k \rightarrow \infty}\left\|T_{i}^{m_{k+1}} x_{k}-T_{i}^{m_{k}} x_{k}\right\|=0$ and $\lim _{\mathcal{K}_{i} \ni k \rightarrow \infty} T_{i}^{m_{k+1}} x_{k}=x^{*}$, i.e., $\lim _{\mathcal{K}_{i} \ni k \rightarrow \infty} T_{i}\left(T_{i}^{m_{k+1}-1} x_{k}\right)=x^{*}$. It then follows that, for each $i \geq 1$,

$$
\lim _{\mathcal{K}_{i} \ni k \rightarrow \infty} T_{i}\left(T_{i}^{m_{k}} x_{k}\right)=x^{*}, \quad \forall i \geq 1 .
$$

In view of the closedness of $T_{i}$, it follows from (3.12) that $T_{i} x^{*}=x^{*}$, i.e., for each $i \geq 1$, $x^{*} \in F\left(T_{i}\right)$ and hence $x^{*} \in F$.

(V) $x^{*}$ is also a member of $G$.

Since $x_{n+1}=\Pi_{C_{n+1}} x_{1}$, it follows from (3.1) and (3.6) that

$$
\phi\left(x_{k+1}, u_{k}\right) \leq \phi\left(x_{k+1}, x_{k}\right)+\xi_{k} \rightarrow 0 \quad\left(\mathcal{K}_{i} \ni k \rightarrow \infty\right) .
$$

Since $x_{k} \rightarrow x^{*}$ as $\mathcal{K}_{i} \ni k \rightarrow \infty$, by virtue of Lemma 2.1 we have

$$
\lim _{\mathcal{K}_{i} \ni k \rightarrow \infty} u_{k}=x^{*}, \quad \forall i \geq 1
$$

This, together with (3.10), shows that $\lim _{\mathcal{K}_{i} \ni k \rightarrow \infty}\left\|u_{k}-y_{k}\right\|=0$ and $\lim _{\mathcal{K}_{i} \ni k \rightarrow \infty} \| J u_{k}-$ $J y_{k} \|=0$. By the assumption that $\left\{r_{k}\right\}_{k \in \mathcal{K}_{i}} \subset[a, \infty)$ for some $a>0$, we have

$$
\lim _{\mathcal{K}_{i} \ni k \rightarrow \infty} \frac{\left\|J u_{k}-J y_{k}\right\|}{r_{k}}=0, \quad \forall i \geq 1 .
$$


Since $\tau\left(u_{k}, y\right)+\frac{1}{r_{k}}\left\langle y-u_{k}, J u_{k}-J y_{k}\right\rangle \geq 0, \forall y \in C$, by condition $\left(\mathrm{A}_{1}\right)$, we have

$$
\frac{1}{r_{k}}\left\langle y-u_{k}, J u_{k}-J y_{k}\right\rangle \geq-\tau\left(u_{k}, y\right) \geq \tau\left(y, u_{k}\right), \quad \forall y \in C .
$$

By the assumption that the mapping $y \mapsto \tau(x, y)$ is convex and lower semicontinuous, letting $\mathcal{K}_{i} \ni k \rightarrow \infty$ in (3.17), from (3.15) and (3.16), we have $\tau\left(y, x^{*}\right) \leq 0, \forall y \in C$. For any $t \in(0,1]$ and any $y \in C$, set $y_{t}=t y+(1-t) x^{*}$. Then $\tau\left(y_{t}, x^{*}\right) \leq 0$ since $y_{t} \in C$. By conditions $\left(\mathrm{A}_{1}\right)$ and $\left(\mathrm{A}_{4}\right)$, we have

$$
0=\tau\left(y_{t}, y_{t}\right) \leq t \tau\left(y_{t}, y\right)+(1-t) \tau\left(y_{t}, x^{*}\right) \leq t \tau\left(y_{t}, y\right)
$$

Dividing both sides of the above equation by $t$, we have $\tau\left(y_{t}, y\right) \geq 0, \forall y \in C$. Letting $t \downarrow 0$, from condition $\left(\mathrm{A}_{3}\right)$, we have $\tau\left(x^{*}, y\right) \geq 0, \forall y \in C$, i.e., $x^{*} \in \Omega$ and so $x^{*} \in G$.

(VI) $x^{*}=\Pi_{G} x_{1}$, and so $x_{n} \rightarrow \Pi_{G} x_{1}$ as $n \rightarrow \infty$.

Put $u=\Pi_{G} x_{1}$. Since $u \in G \subset C_{n}$ and $x_{n}=\Pi_{C_{n}} x_{1}$, we have $\phi\left(x_{n}, x_{1}\right) \leq \phi\left(u, x_{1}\right), \forall n \geq 1$. Then

$$
\phi\left(x^{*}, x_{1}\right)=\lim _{n \rightarrow \infty} \phi\left(x_{n}, x_{1}\right) \leq \phi\left(u, x_{1}\right)
$$

which implies that $x^{*}=u$ since $u=\Pi_{G x_{1}}$, and hence $x_{n} \rightarrow x^{*}=\Pi_{F} x_{1}$ as $n \rightarrow \infty$. This completes the proof.

We now provide a nontrivial family of mappings satisfying the conditions of Theorem 3.1.

Example 3.2 Let $E=\mathbb{R}^{1}$ with the standard norm $\|\cdot\|=|\cdot|$ and $C=[-1,1]$. Let $\left\{T_{i}\right\}: C \rightarrow C$ be a countable family of mappings defined by

$$
T_{i} x= \begin{cases}\frac{-x}{i^{2}+1}, & x \in(0,1] \\ x, & x \in[-1,0] .\end{cases}
$$

We first show that $\left\{T_{i}\right\}$ is uniformly $L$-Lipschitzian. If $x \in(0,1]$ and $y \in[-1,0]$, then

$$
\left|T_{i}^{n} x-T_{i}^{n} y\right|=\left|\frac{(-1)^{n}}{\left(i^{2}+1\right)^{n}} x-y\right| \leq|x|+|y| \leq 2|x-y| .
$$

The rest is trivial. Second, we claim that $\left\{T_{i}\right\}$ is a family of closed and totally quasi- $\phi$ asymptotically nonexpansive mappings. In fact, for any $x \in(0,1]$ and $p \in \bigcap_{i=1}^{\infty} F\left(T_{i}\right)=$ $[-1,0]$, we have, for all $n \geq 2$,

$$
\begin{aligned}
\phi\left(p, T_{i}^{n} x\right) & =\left|T_{i}^{n} x-p\right|^{2}=\left|\frac{(-1)^{n}}{\left(i^{2}+1\right)^{n}} x-p\right|^{2} \\
& \leq\left[|p|+\frac{1}{\left(i^{2}+1\right)^{n}}|x|\right]^{2} \\
& \leq\left[|x-p|+\frac{1}{\left(i^{2}+1\right)^{n}}\right]^{2} \\
& =\phi(x, p)+v_{n}^{(i)} \zeta(\phi(x, p))+\mu_{n}^{(i)},
\end{aligned}
$$


where $\phi(x, p)=|x-p|^{2}, v_{n}^{(i)}=\frac{2}{\left(i^{2}+1\right)^{n}}, \mu_{n}^{(i)}=\frac{1}{\left(i^{2}+1\right)^{2 n}}$ and $\zeta(x)=\sqrt{x}$. Note that $\left|T_{i} x-p\right|^{2} \leq$ $|x-p|^{2}$, that is, $\mu_{1}^{(i)}=0$ for each $i \geq 1$.

Next, we define a bifunction $\theta: C \times C \rightarrow \mathbb{R}$ satisfying the conditions $\left(\mathrm{A}_{1}\right)-\left(\mathrm{A}_{4}\right)$ by

$$
\theta(x, y)=y^{2}-x^{2}
$$

Let $A=0$ and $\psi=0$. Then the set of solutions $\Omega$ to the generalized mixed equilibrium problem for $\theta, A$ and $\psi$ is obviously $\{0\}$. Since $G:=\Omega \cap F \neq \emptyset$ and $F$ is bounded, it follows from Theorem 3.1 that the sequence $\left\{x_{n}\right\}$ defined by (3.1) converges strongly to $\Pi_{G} x_{1}$.

\section{Competing interests}

The author declares that they have no competing interests.

\section{Acknowledgements}

The author is very grateful to the referees for their useful suggestions, by which the contents of this article has been improved. This study is supported by the National Natural Science Foundation of China (Grant No. 11061037) and the General Project of Scientific Research Foundation of Yunnan University of Finance and Economics (YC2013A02).

\section{Received: 20 August 2013 Accepted: 28 February 2014 Published: 14 Mar 2014}

\section{References}

1. Chang, SS, Lee, HWJ, Chan, CK, Yang, L: Approximation theorems for total quasi- $\phi$-asymptotically nonexpansive mappings with applications. Appl. Math. Comput. 218, 2921-2931 (2011). doi:10.1016/j.amc.2011.08.036

2. Chang, SS, Lee, HWJ, Chan, CK, Zhang, WB: A modified Halpern-type iterative algorithm for totally quasi- $\phi$-asymptotically nonexpansive mappings with applications. Appl. Math. Comput. 218, 6489-6497 (2012) doi:10.1016/j.amc.2011.12.019

3. Matsushita, S, Takahashi, W: A strong convergence theorem for relatively nonexpansive mappings in Banach spaces. J. Approx. Theory 134, 257-266 (2005). doi:10.1016/j.jat.2005.02.007

4. Plubtieng, S, Ungchittrakool, K: Hybrid iterative methods for convex feasibility problems and fixed point problems of relatively nonexpansive mappings in Banach spaces. Fixed Point Theory Appl. 2008, Article ID 583082 (2008). doi:10.1155/2008/583082

5. Chang, SS, Lee, HWJ, Chan, CK: A block hybrid method for solving generalized equilibrium problems and convex feasibility problem. Adv. Comput. Math. (2011). doi:10.1007/s10444-011-9249-5

6. Ceng, LC, Guu, SM, Hu, HY, Yao, JC: Hybrid shrinking projection method for a generalized equilibrium problem, a maximal monotone operator and a countable family of relatively nonexpansive mappings. Comput. Math. Appl. 61 2468-2479 (2011). doi:10.1016/j.camwa.2011.02.028

7. Su, YF, Xu, HK, Zhang, X: Strong convergence theorems for two countable families of weak relatively nonexpansive mappings and applications. Nonlinear Anal. 73, 3890-3906 (2010). doi:10.1016/j.na.2010.08.021

8. Ofoedu, EU, Malonza, DM: Hybrid approximation of solutions of nonlinear operator equations and application to equation of Hammerstein-type. Appl. Math. Comput. 217, 6019-6030 (2011). doi:10.1016/j.amc.2010.12.073

9. Wang, ZM, Su, YF, Wang, DX, Dong, YC: A modified Halpern-type iteration algorithm for a family of hemi-relative nonexpansive mappings and systems of equilibrium problems in Banach spaces. J. Comput. Appl. Math. 235, 2364-2371 (2011). doi:10.1016/j.cam.2010.10.036

10. Chang, SS, Chan, CK, Lee, HWJ: Modified block iterative algorithm for quasi- $\phi$-asymptotically nonexpansive mappings and equilibrium problem in Banach spaces. Appl. Math. Comput. 217, 7520-7530 (2011). doi:10.1016/j.amc.2011.02.060

11. Yao, YH, Liou, YC, Kang, SM: Strong convergence of an iterative algorithm on an infinite countable family of nonexpansive mappings. Appl. Math. Comput. 208, 211-218 (2009). doi:10.1016/j.amc.2008.11.038

12. Zegeye, H, Ofoedu, EU, Shahzad, N: Convergence theorems for equilibrium problem, variational inequality problem and countably infinite relatively quasi-nonexpansive mappings. Appl. Math. Comput. 216, 3439-3449 (2010). doi:10.1016/j.amc.2010.02.054

13. Nilsrakoo, W, Saejung, S: Strong convergence theorems by Halpern-Mann iterations for relatively non-expansive mappings in Banach spaces. Appl. Math. Comput. 217, 6577-6586 (2011). doi:10.1016/j.amc.2011.01.040

14. Chang, SS, Lee, HWJ, Chan, CK, Liu, J: Strong convergence theorems for countable families of asymptotically relatively nonexpansive mappings with applications. Appl. Math. Comput. 218, 3187-3198 (2011). doi:10.1016/j.amc.2011.08.055

15. Zhang, S-s: Generalized mixed equilibrium problem in Banach space. Appl. Math. Mech. 30, 1105-1112 (2009). doi:10.1007/s10483-009-0904-6

16. Chang, SS, Kim, JK, Wang, XR: Modified block iterative algorithm for solving convex feasibility problems in Banach spaces. J. Inequal. Appl. 2010, Article ID 869684 (2010). doi:10.1155/2010/869684

17. Luchuan, $C$, Jenchih, Y: A hybrid iterative scheme for mixed equilibrium problems and fixed point problems. J. Comput. Appl. Math. 214, 186-201 (2008). doi:10.1016/j.cam.2007.02.022

18. Qin, X, Cho, SY, Kang, SM: Strong convergence of shrinking projection methods for quasi- $\boldsymbol{\phi}$-nonexpansive mappings and equilibrium problems. J. Comput. Appl. Math. 234(3), 750-760 (2010) 
19. Saewan, S, Kumam, P: A modified hybrid projection method for solving generalized mixed equilibrium problems and fixed point problems in Banach spaces. Comput. Math. Appl. 62(4), 1723-1735 (2011)

20. Alber, Yl: Metric and generalized projection operators in Banach spaces: properties and applications. In: Kartsatos, AG (ed.) Theory and Applications of Nonlinear Operators of Accretive and Monotone Type, pp. 15-50. Dekker, New York (1996)

21. Cioranescu, I: Geometry of Banach Spaces, Duality Mappings and Nonlinear Problems. Kluwer Academic, Dordrecht (1990)

22. Chang, SS, Wang, L, Tang, YK, Zhao, YH, Ma, ZL: Strong convergence theorems of nonlinear operator equations for countable family of multi-valued total quasi- $\phi$-asymptotically nonexpansive mappings with applications. Fixed Point Theory Appl. 2012, 69 (2012). doi:10.1186/1687-1812-2012-69

23. Deng, WQ, Bai, P: An implicit iteration process for common fixed points of two infinite families of asymptotically nonexpansive mappings in Banach spaces. J. Appl. Math. 2013, Article ID 602582 (2013)

10.1186/1687-1812-2014-63

Cite this article as: Deng: A relaxed hybrid shrinking iteration approach to solving generalized mixed equilibrium problems for totally quasi- $\phi$-asymptotically nonexpansive mappings. Fixed Point Theory and Applications 2014, 2014:63

\section{Submit your manuscript to a SpringerOpen ${ }^{\circ}$ journal and benefit from:}

- Convenient online submission

- Rigorous peer review

- Immediate publication on acceptance

Open access: articles freely available online

- High visibility within the field

- Retaining the copyright to your article 